\title{
ECOLOGICAL FEATURES OF CENTAUREA L. SECTION PHALOLEPIS (CASS.) DC. IN TURKEY
}

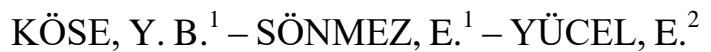 \\ ${ }^{1}$ Anadolu University, Faculty of Pharmacy, Department of Pharmaceutical Botany, 26470 \\ Eskisehir \\ ${ }^{2}$ Anadolu University, Faculty of Science, Department of Biology, 26470 Eskisehir \\ *Corresponding author \\ e-mail:ybkose@anadolu.edu.tr \\ (phone: +90222 3350580) \\ (Received $7^{\text {th }}$ Jul 2016; accepted 26 $6^{\text {th }}$ Sep 2016)
}

\begin{abstract}
In this present study, the ecological characteristics of Phalolepis (Cass.) DC. a section of the Centaurea L. genus growing in Turkish was investigated. Important to note is that all species of the section are restricted endemics. Physico-chemical soil analyses as well as chemical analyses of different parts of the species were conducted. Soil and bedrock samples were investigated resuling in petrographic diagnoses. Discriminant analyses were used to determine the amount of the plant nutritional elements of the different plant parts such as root, stem, and leaf, depending on physical and chemical features of 0-10 $\mathrm{cm}$ soil specimens. The amount of the nutrient elements in the roots, stem and leaves, the classification success percentages were 92.3, 92.3 and 88.5 , respectively. The discrimination analysis success percentage of the chemical and physical soil contents resulted in $96.2 \%$.
\end{abstract}

Keywords: ecology, Centaurea, soil, plant, analysis

\section{Introduction}

Ecological areas are the result of geological, paleogeographic and historical factors where protected areas are determined based on endemic species (Olson and Dinerstein, 1998; Mittermaier et al., 1998; WWF and IUCN 1994-2001). Islands, mountains or isolated edaphic systems such as ultrabasic rocks, gypsum or limestone areas are generally classified as endemic plant areas (Favarger, 1972; Gomez-Campo et al., 1984). Widespread species become rare depending on the effects of humans on nature. Naturally rare and aquianted rare species are not distinguished easily (Rabinowitz et al, 1986; Gaston, 1994; Norton and De Lange, 1998). Restricted areas such as rocky slopes and cliffs are one of the major reasons for naturally rare plant species (Gaston, 1994). This kind of natural isolations and extreme habitats may lead to formations of local endemic and naturally rare plant species (Wyatt, 1997; Larson et al., 2000; Hopper, 2000). Geographical locations, ecological characteristics, paleogeography and historical features make Turkey rich for its flora. Although Turkey is about one fifth of Europe it has relatively more endemic plant species when compared. Approximately 30\% of Turkish Flora is endemic, while all Europe consists about 2750 endemic plants (Ekim et al., 2000).

Globally around 700 Centaurea L. species are present in Asia, North Africa and America (Brummitt, 2004). The genus Centaurea is represented in Turkey by 34 sections, 226 species and the overall endemism rate is $66 \%$ (Kültür, 2016). All of the Phalolepis section species are restricted endemic species, one of them is classified as NT (Near Threatened), 4 species are EN (ENdangered), and 4 species are CR (Critical Endangered) (IUCN, 2001). 
The main purpose of the study was to determine ecological features of the section Phalolepis.

\section{Materials and Methods}

Studied species were collected from their natural habitats between 2003-2005 (see Table 1, Fig. 1). Petrographic diagnoses were made both for soil and bedrock samples of plant species. Geological features of all section members were investigated by comparing Turkey geological map (1/500 000 scales), which was previously prepared by the Mineral Research and Exploration Institute (Duberted, 1973).

Plant samples of the root, stem and leaves were evaluated in detail, respectively. Chemical analyses were made by Semi-Micro Kjeldhal method for N (Jackson, 1962), Olsen method for P (Chapman and Pratt, 1961); Ca, Mg, K, ammonium-acetate; Na, sodium-acetate (Jackson, 1962); Fe, Cu, Zn and Mn wet digestion method (Walkley and Black, 1934). Also, 0-10 cm depth soil samples of the plants were collected from the areas, which were dried and color of the samples were compared with Standart Soil Color Charts (1970). Physical analyses of soils were made by Bouyoucus hydrometer method (1962), and soil types were determined using soil types utilizing triangle (Çepel, 1983). Chemical analyses of soils were made with Beckman and $\mathrm{pH}$ meter about 0,01 sensitivity (Jackson, 1962). Soil reaction was observed the in $1 / 2.5$ ratio of suspension, the values of soil $\mathrm{pH}$ were compared with Kantarc1 (2000). The amount of salt in the soil was determined by Conductance Bridge device (Jackson, 1962), and the results were compared with Eruz (1979). Amounts of $\mathrm{CaCO}_{3}$ of soils were determined with Scheibler (Çepel, 1983), and the results were compared with Tüzüner (1990). Total N amounts of soils were determined by micro Kjeldahl Method (Jackson, 1962), and compared with Schröder (1972). Organic soil contents and $\mathrm{Fe}, \mathrm{Cu}, \mathrm{Zn}$ and $\mathrm{Mn}$ analyses were determined by the combustion method of Walkley and Black (1934). P amount was determined by Olsen method (Chapman and Pratt, 1961). Soil extracts were prepared for ammonium-acetate method (Jackson, 1962), and the results were evaluated comparing with Schröder (1972). Discrimination method was used for plant nutrient elements and soil types. Statistical analyses were performed using SPSS 10.0 software.

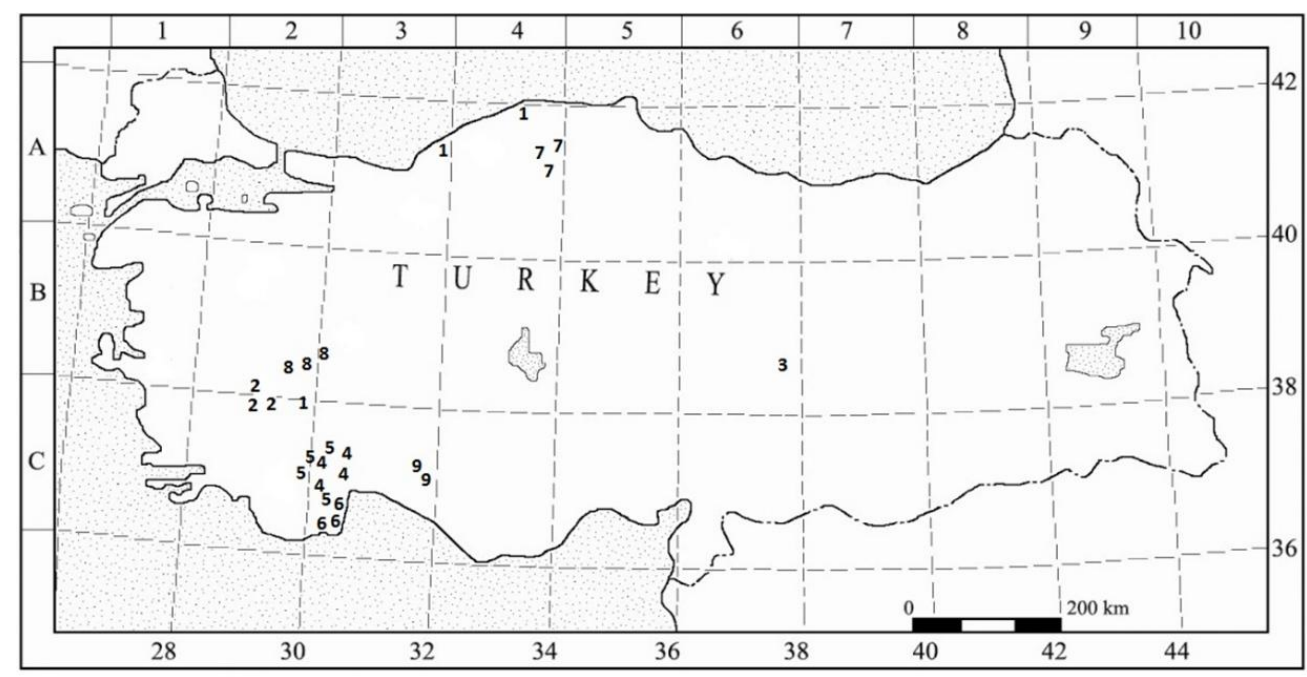

Figure 1. Study fields 
Table 1. The localities of Phalolepis section

\begin{tabular}{|c|c|c|c|}
\hline & Species & $\begin{array}{c}\text { Sample } \\
\text { area }\end{array}$ & Locality \\
\hline & & 1 & Denizli: Honaz, National Park way, cliff, 804 m, 24 vi 2004, K 37º 44' 58.2”, D $29^{\circ} 16^{\prime} 07.3^{\prime \prime}$ \\
\hline \multirow[t]{3}{*}{1} & C. cadmea & 2 & Zonguldak: Devrek-Eğerci way, Yeşilöz village, cliff, 330 m, 19 vi $2004, \mathrm{~K} 41^{\circ} 05^{\prime} 42.4^{\prime \prime}, \mathrm{D} 31^{\circ} 50^{\prime} 06.2^{\prime \prime}$ \\
\hline & & 3 & Bartın: Ulus, Ulukaya waterfall, cliff, 275 m., 6 ix $2005, \mathrm{~K} 41^{\circ} 40^{\prime} 07.4^{\prime \prime}$, D $32^{\circ} 45^{\prime} 59.8^{\prime \prime}$ \\
\hline & & 1 & Aydın/Denizli: Geyre-Tavas way, rood side, rocky slopes, 1022 m, 25 vi 2004 , K $37^{0} 39^{\prime} 53.0^{\prime \prime}$ D $28^{0} 51^{\prime} 52.7^{\prime \prime}$ \\
\hline \multirow[t]{2}{*}{2} & C. aphrodisea & 2 & İzmir: Ödemiş, Bozdağ, Ski center road, cliff, 1200 m, 25 vii $2004, \mathrm{~K} 38^{0} 21^{\prime} 07.8^{\prime \prime} \mathrm{D} 28^{0} 05^{\prime} 19.6 ”$ \\
\hline & & 3 & Denizli: Başkarcı village, İsrail waterfall, Picnic area, rocky slopes, 933 m, K $37^{0} 55^{\prime} 42.6^{\prime \prime}$ D $29^{0} 08^{\prime} 07.4^{\prime \prime}$ \\
\hline \multirow[t]{2}{*}{3} & C. amaena & 1 & Kayseri: Exit of Y1lanlı mountain, rood side, cliff, 1194 m, 14 vii 2004, N 380 42' 55.4” E 350 25' 18.2 "' \\
\hline & & 1 & Antalya: Antalya-Korkuteli way, $20 . \mathrm{km}$, rood side, cliff, $538 \mathrm{~m}, 2$ vi 2003 , K $37^{0} 01$ ' $35.7^{\prime \prime} \mathrm{D} 30^{\circ} 27^{\prime} 39.6$ " \\
\hline \multirow[t]{5}{*}{4} & & 2 & Antalya: Saklıkent way, $9 \mathrm{~km}$ before the plants, rocky slopes, $1142 \mathrm{~m}, 5$ vii 2003 \\
\hline & C. lycıa & 3 & Antalya: Kozdağı, Tahtalı resting place road, rocky slopes, $1130 \mathrm{~m}, 5$ vii 2003 , K $36^{0} 53^{\prime} 51.5^{\prime \prime} \mathrm{D} 30^{\circ} 22^{\prime} 21.5^{\prime \prime}$ \\
\hline & & 4 & Burdur: Kizllkaya-Korkuteli way, Steep cliff, 844 m, 2 vii 2005 , K $36^{0} 18^{\prime} 32.6^{\prime \prime}$ D $30^{0} 21^{\prime} 26.9^{\prime}$ ' \\
\hline & & 1 & Antalya: Between Elmal1-Korkuteli, Karaman beli, cliff, 1300 m, 5 vii 2003 , K $36^{\circ} 56^{\prime} 52.5^{\prime \prime}$ D $30^{0} 09^{\prime} 43.8^{\prime \prime}$ \\
\hline & & 2 & Antalya: Elmalı-Korkuteli way, rood side, limestone rocks, 1156 m, 4 vii 2003 , K $36^{0} 45^{\prime} 09.6^{\prime \prime}$ D $29^{0} 54^{\prime} 22.6^{\prime \prime}$ \\
\hline \multirow[t]{3}{*}{5} & C. luschaniana & 3 & Antalya: Between Korkuteli-Elmalı, 30. km, 1308 m, 4 vii 2003, K $36^{0} 56^{\prime} 37.7^{\prime \prime}$ D $30^{0} 07^{\prime} 04.4 ”$ \\
\hline & & 4 & Antalya: Between Korkuteli-Elmalı, 14. km, cliff, 1265 m, 3 vii 2005, K $36^{0} 58^{\prime} 17.3^{\prime \prime}$ D $30^{\circ} 09^{\prime} 05.7^{\prime \prime}$ \\
\hline & & 1 & Antalya: Adrasan, Sazak way, under red pine, 18 m, 23 v 2004, K $36^{\circ} 18^{\prime} 52.4^{\prime \prime}$ D $30^{\circ} 28^{\prime} 00.0 "$ \\
\hline \multirow[t]{3}{*}{6} & C. wagenitzii & 2 & Antalya: Adrasan, southwest of cost, walkways, macchia, $3 \mathrm{~m}, 9$ vi 2004 , K $36^{\circ} 17^{\prime} 53.8^{\prime \prime} \mathrm{D} 30^{\circ} 28^{\prime} 25.6 "$ \\
\hline & & 3 & Antalya: Adrasan, southwest slopes, macchia, $13 \mathrm{~m}, 3$ vii 2005 , $\mathrm{K} 36^{\circ} 17^{\prime} 54.1^{\prime \prime}$ D $30^{\circ} 28^{\prime} 26.5^{\prime \prime}$ \\
\hline & & 1 & Kastamonu: Between Tosya-Kastamonu, roodside, open forest area, $1048 \mathrm{~m}, 5$ ix 2005 , K $41^{\circ} 11^{\prime} 25.0^{\prime \prime}$ D $34^{0} 01^{\prime} 40.7^{\prime \prime}$ \\
\hline \multirow[t]{3}{*}{7} & C. tossiensis & 2 & Kastamonu: Daday, between Hasanağa-Çayözü, stony slope, 1035 m, 5 ix $2005, \mathrm{~K} 41^{0} 35^{\prime} 05.7^{\prime \prime}$ D $33^{0} 30^{\prime} 00.7^{\prime \prime}$ \\
\hline & & 3 & Kastamonu: between Kastamonu-Araç, Ahlatçık village road, forest clearance, 1154 m, 6 ix 2005 \\
\hline & & 1 & Afyon: Between Dazkırı-Çardak, Sarıkavak village, pond side, 974 m, 2 vii 2003, N $37^{0} 533^{\prime} 29.4^{\prime \prime}$ E $29^{0} 48^{\prime} 32.4^{\prime \prime}$ \\
\hline \multirow[t]{2}{*}{8} & C. hieropolitana & 2 & Denizli: Pamukkale, The front of travertine, 318 m, 24 vi 2004, N $37^{0} 55^{\prime} 20.4 "$ E $29^{0} 07^{\prime} 00.7 "$ \\
\hline & & 3 & 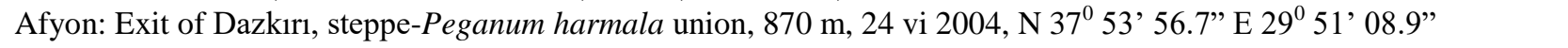 \\
\hline \multirow[b]{2}{*}{9} & & 1 & Antalya: Akseki, Güzelsu way, Serebel well side, under P. brutia, 1090 m, 6 vii 2003 \\
\hline & C. antalyense & 2 & Antalya: Sadıklar-Güzelsu way, under Cedrus, $1077 \mathrm{~m}, 3$ vii 2055 , K $36^{0} 54^{\prime} 46.1^{\prime \prime} \mathrm{D} 31^{0} 48^{\prime} 48.3$ ', \\
\hline
\end{tabular}




\section{Results and Discussion}

\section{Bedrock and Geology}

Sample area of $C$. cadmea in Denizli-Honaz, bedrock is metamorphic schist while the geological structure is paleozoic; in Zonguldak-Devrek-Eğerci, main rock is andesite, the geological structure is Eocene, Paleocene, and Cretaceous; in Bartın-Ulus, bedrock is limestone and the geological structure is Cretaceous. Sample area of $C$. aphrodisea in Ödemiş-Bozdağ, bedrock is mica schist while the geological structure is paleozoic; in Aydin-Geyre, bedrock is mica schist, the geological structure is Neogene; in Denizli-Başkarc1, bedrock is crystallized limestone and the geological structure is paleozoic. Sample area of $C$. amaena in Kayseri-Y1lanlı Mountain, bedrock is agglomerate and the geological structure is paleozoic. Sample areas of C. lycia in Antalya-Korkuteli road, Antalya-Kozdağı, Burdur-Kızılkaya and Antalya-Saklıkent main rocks are limestones and the geological structures are Mesozoic-tertiary in Antalya-Korkuteli road, Antalya-Kozdağı, and Antalya-Saklıkent. The geological structure of Burdur-Kizilkaya is Holocene. All sample areas of $C$. luschaniana main rocks are limestones, in Karaman beli, between Elmali-Korkuteli $30 \mathrm{~km}$ and $14 \mathrm{~km}$ geological structures are Holocene, and in the other locality geological structure is Miocene. In three localities of $C$. wagenitzii bedrocks are alluvial, and the geological structures are Quaternary. Sample area of $C$. tossiensis on rood side, bedrock is serpentine, the geological structure is Eocene; in Tosya bedrock is ultrabasic rocks, the geological structure is Neogene; in Daday bedrock is ultrabasic rocks and the geological structure is Cretaceous. Sample area of C. hieropolitana in Sarıkavak village between Dazkırı-Çardak main rock is marl, the geological structure is Oligocene; in the exit of Dazkırı bedrock is limestone, the geological structure is Quaternary; in DenizliPamukkale bedrock is travertine and the geological structure is paleozoic. Sample areas of $C$. antalyense in Güzelsu-Serebel well side and Sadıklar-Güzelsu way both two localities have limestone bedrocks and upper Cretaceous as the geological structures.

\section{Plant Analysis}

The amounts of nutrient elements of plants roots are seen in Table 2. The highest amount of $\mathrm{N}$ have been found $3.1351 \%$ and is seen on the second sample area of $C$. cadmea. The lowest one is $0.3327 \%$ and is seen on the fourth sample area of $C$. luschaniana. The highest $\mathrm{Mg}$ rate $(10250 \mathrm{ppm})$ is seen on the first sample area of $C$. aphrodisea and the highest $\mathrm{Zn}$ amount $(419.5 \mathrm{ppm})$ is seen on the first sample area of $C$. luschaniana.

The amounts of nutrient elements of the plants stems has given in Table 3. The analyses show the stems have lower $\mathrm{N}$ amounts than the roots. A third sample area of $C$. wagenitzii, second sample area of $C$. tossiensis and third sample area of $C$. hieropolitana stems have higher $\mathrm{N}$ rates than the roots. A third sample area of $C$. wagenitzii has the highest $\mathrm{Na}(1300 \mathrm{ppm})$, third sample area of $C$. cadmea has the highest $\mathrm{Fe}(2500 \mathrm{ppm})$, first sample area of $C$. lycia has the highest $\mathrm{Cu}(125.5 \mathrm{ppm})$ rates. 
Table 2. The nutrient elements of species roots

\begin{tabular}{|c|c|c|c|c|c|c|c|c|c|c|c|}
\hline Species & Locality & $\begin{array}{l}\mathrm{N} \\
\%\end{array}$ & $\begin{array}{c}\mathrm{Na} \\
\mathrm{ppm}\end{array}$ & $\begin{array}{c}\mathrm{Mg} \\
\mathrm{ppm}\end{array}$ & $\begin{array}{c}\mathrm{Ca} \\
\mathrm{ppm}\end{array}$ & $\begin{array}{c}\mathrm{Fe} \\
\mathrm{ppm}\end{array}$ & $\begin{array}{c}\mathrm{K} \\
\mathrm{ppm}\end{array}$ & $\begin{array}{c}\text { Mn } \\
\text { ppm }\end{array}$ & $\begin{array}{c}\mathrm{Zn} \\
\mathrm{ppm}\end{array}$ & $\begin{array}{c}\mathrm{Cu} \\
\mathrm{ppm}\end{array}$ & $\begin{array}{c}\mathrm{P} \\
\mathrm{ppm}\end{array}$ \\
\hline \multirow{4}{*}{ C. cadmea } & 1 & 1,0603 & 400 & 1715 & 31795 & 2130 & 15000 & 167,5 & 39 & 30 & 1550 \\
\hline & 2 & 3,1351 & 450 & 1555 & 14525 & 1430 & 11000 & 398,5 & 71 & 29,5 & 550 \\
\hline & 3 & 1,0564 & 700 & 1595 & 29760 & 2310 & 15250 & 140,5 & 31,5 & 31,5 & 1650 \\
\hline & 1 & 1,3938 & 225 & 10250 & 9500 & 2970 & 6250 & 152 & 38 & 15,5 & 525 \\
\hline \multirow[t]{2}{*}{ C. aphrodisea } & 2 & 1,8704 & 575 & 1335 & 8680 & 1080 & 13500 & 96,5 & 10,5 & 11 & 450 \\
\hline & 3 & 0,7167 & 450 & 1635 & 25620 & 1920 & 7000 & 209 & 57 & 18 & 700 \\
\hline \multirow[t]{2}{*}{ C. amaena } & 1 & 1,4651 & 350 & 1185 & 21450 & 445 & 8000 & 77 & 71 & 30 & 950 \\
\hline & 1 & 2,0476 & 1500 & 1250 & 17590 & 445 & 15000 & 43,5 & 90 & 33,5 & 1550 \\
\hline \multirow{3}{*}{ C. lycia } & 2 & 1,1029 & 575 & 1175 & 4740 & 680 & 7250 & 82 & 72,5 & 20 & 1050 \\
\hline & 3 & 1,4509 & 625 & 1010 & 7915 & 215 & 10500 & 14 & 72 & 9 & 900 \\
\hline & 4 & 1,2018 & 625 & 2130 & 22805 & 3995 & 11000 & 154,5 & 37 & 20,5 & 1300 \\
\hline \multirow{5}{*}{ C. luschaniana } & 1 & 1,1653 & 775 & 1780 & 13495 & 2025 & 10500 & 113,5 & 419,5 & 37 & 1250 \\
\hline & 2 & 0,8293 & 600 & 940 & 8675 & 980 & 8750 & 90 & 84 & 44 & 700 \\
\hline & 3 & 0,969 & 350 & 995 & 14485 & 1335 & 6250 & 91 & 80,5 & 56,5 & 950 \\
\hline & 4 & 0,3327 & 575 & 1135 & 15300 & 520 & 8750 & 42,5 & 18,5 & 13,5 & 450 \\
\hline & 1 & 2,0484 & 875 & 6695 & 16535 & 1520 & 7000 & 74 & 174 & 22 & 2100 \\
\hline \multirow[t]{2}{*}{ C. wagenitzii } & 2 & 0,3576 & 550 & 1455 & 3750 & 900 & 5000 & 22 & 31 & 14,5 & 200 \\
\hline & 3 & 0,6382 & 775 & 1155 & 2865 & 330 & 6250 & 8,5 & 20,5 & 10 & 525 \\
\hline \multirow{3}{*}{ C. tossiensis } & 1 & 0,9056 & 350 & 1310 & 9250 & 350 & 13000 & 38 & 26,5 & 15 & 2350 \\
\hline & 2 & 0,4396 & 275 & 1450 & 8455 & 235 & 12500 & 20 & 16,5 & 9 & 1000 \\
\hline & 3 & 0,5862 & 350 & 1305 & 10640 & 585 & 11750 & 79,5 & 37,5 & 16,5 & 1100 \\
\hline \multirow{3}{*}{ C. hieropolitana } & 1 & 0,9151 & 1050 & 1470 & 9380 & 500 & 10000 & 21,5 & 69,5 & 11 & 250 \\
\hline & 2 & 1,2687 & 400 & 6550 & 35005 & 500 & 14500 & 31 & 68,5 & 13 & 750 \\
\hline & 3 & 1,592 & 1175 & 1795 & 16575 & 1035 & 9500 & 39 & 53,5 & 6,5 & 525 \\
\hline \multirow{2}{*}{ C. antalyense } & 1 & 1,0647 & 675 & 1435 & 12850 & 1870 & 19500 & 85,5 & 176 & 33,5 & 1850 \\
\hline & 2 & 0,8107 & 500 & 1080 & 10400 & 1290 & 10000 & 56 & 29 & 26,5 & 1350 \\
\hline
\end{tabular}

Table 3. The nutrient elements of species stems

\begin{tabular}{|c|c|c|c|c|c|c|c|c|c|c|c|}
\hline Species & Locality & $\begin{array}{l}\mathrm{N} \\
\%\end{array}$ & $\begin{array}{c}\mathrm{Na} \\
\mathrm{ppm}\end{array}$ & $\begin{array}{c}\mathrm{Mg} \\
\mathrm{ppm}\end{array}$ & $\begin{array}{c}\mathrm{Ca} \\
\mathrm{ppm}\end{array}$ & $\begin{array}{c}\mathrm{Fe} \\
\mathrm{ppm}\end{array}$ & $\begin{array}{c}\mathrm{K} \\
\mathrm{ppm}\end{array}$ & $\begin{array}{c}\mathrm{Mn} \\
\mathrm{ppm}\end{array}$ & $\begin{array}{c}\mathrm{Zn} \\
\mathrm{ppm}\end{array}$ & $\begin{array}{c}\mathrm{Cu} \\
\mathrm{ppm}\end{array}$ & $\begin{array}{c}\mathrm{P} \\
\mathrm{ppm}\end{array}$ \\
\hline \multirow{4}{*}{ C. cadmea } & 1 & 1,0048 & 275 & 1215 & 13150 & 445 & 10500 & 24 & 17,5 & 9,5 & 1600 \\
\hline & 2 & 1,1763 & 300 & 1040 & 8230 & 270 & 14500 & 106,5 & 19 & 9 & 750 \\
\hline & 3 & 0,5922 & 450 & 1340 & 18875 & 2510 & 7250 & 74,5 & 27,5 & 12 & 1600 \\
\hline & 1 & 0,9026 & 500 & 1435 & 11590 & 210 & 9250 & 15 & 18,5 & 5,5 & 1000 \\
\hline \multirow[t]{2}{*}{ C. aphrodisea } & 2 & 0,9808 & 300 & 1100 & 9420 & 755 & 12000 & 36,5 & 10 & 6,5 & 250 \\
\hline & 3 & 0,5566 & 500 & 930 & 6560 & 160 & 9500 & 20,5 & 24,5 & 10 & 700 \\
\hline \multirow{2}{*}{ C. amaena } & 1 & 1,1115 & 200 & 1005 & 11915 & 145 & 14500 & 23 & 24,5 & 9 & 1250 \\
\hline & 1 & 1,6296 & 625 & 1320 & 15790 & 365 & 10500 & 25 & 101 & 125,5 & 1200 \\
\hline \multirow{3}{*}{ C. lycia } & 2 & 1,4202 & 200 & 1155 & 4790 & 375 & 6500 & 18,5 & 83,5 & 11,5 & 300 \\
\hline & 3 & 1,5348 & 275 & 1350 & 14070 & 280 & 14000 & 9,5 & 63,5 & 12,5 & 750 \\
\hline & 4 & 0,7383 & 550 & 1980 & 22700 & 515 & 8500 & 22 & 16,5 & 7 & 950 \\
\hline \multirow{4}{*}{ C. luschaniana } & 1 & 0,984 & 325 & 1265 & 11850 & 505 & 8750 & 28,5 & 181,5 & 42,5 & 1100 \\
\hline & 2 & 0,6869 & 475 & 1145 & 11835 & 385 & 7000 & 29 & 75,5 & 30,5 & 700 \\
\hline & 3 & 0,7157 & 250 & 755 & 7525 & 280 & 5500 & 15,5 & 93 & 23 & 650 \\
\hline & 4 & 0,325 & 175 & 2330 & 16875 & 210 & 5250 & 13,5 & 20 & 5,5 & 600 \\
\hline \multirow{3}{*}{ C. wagenitzii } & 1 & 1,6964 & 350 & 1340 & 12790 & 575 & 8000 & 30,5 & 177 & 17 & 1050 \\
\hline & 2 & 0,5554 & 800 & 2360 & 5715 & 135 & 6250 & 15,5 & 19,5 & 10,5 & 450 \\
\hline & 3 & 0,4 & 1300 & 1800 & 4890 & 185 & 7000 & 13 & 12,5 & 9,5 & 525 \\
\hline \multirow{3}{*}{ C. tossiensis } & 1 & 0,9855 & 450 & 1515 & 14515 & 275 & 12000 & 20,5 & 24,5 & 9 & 2950 \\
\hline & 2 & 0,646 & 250 & 1380 & 14590 & 75 & 10500 & 7 & 12,5 & 8 & 1350 \\
\hline & 3 & 0,8832 & 125 & 1435 & 10315 & 310 & 11250 & 37 & 33,5 & 12,5 & 1350 \\
\hline \multirow{3}{*}{ C. hieropolitana } & 1 & 0,7041 & 700 & 2230 & 11620 & 145 & 11250 & 9,5 & 50 & 10 & 700 \\
\hline & 2 & 1,1337 & 300 & 6150 & 32445 & 200 & 7000 & 29 & 39,5 & 10,5 & 550 \\
\hline & 3 & 1,6449 & 250 & 2180 & 24905 & 240 & 4750 & 17 & 31 & 7 & 950 \\
\hline \multirow{2}{*}{ C. antalyense } & 1 & 0,746 & 325 & 1090 & 8010 & 95 & 18250 & 4,5 & 109,5 & 15 & 850 \\
\hline & 2 & 0,5797 & 400 & 620 & 5275 & 75 & 17250 & 3 & 18,5 & 8,5 & 1650 \\
\hline
\end{tabular}

Table 4 shows the amount of nutrient elements in the leaves of the species. Almost all the elements are the greater amount of the leaves than the roots and stems. In second 
and third sample areas of $C$. wagenitzii have the highest Na (1650-2000 ppm), and third sample area has the highest $\mathrm{Mg}$ (14 $305 \mathrm{ppm}$ ) rates.

Table 4. The nutrient elements of species leaves

\begin{tabular}{|c|c|c|c|c|c|c|c|c|c|c|c|}
\hline Species & Locality & $\begin{array}{l}\mathrm{N} \\
\%\end{array}$ & $\begin{array}{c}\mathrm{Na} \\
\mathrm{ppm}\end{array}$ & $\begin{array}{c}\mathrm{Mg} \\
\mathrm{ppm}\end{array}$ & $\begin{array}{c}\mathrm{Ca} \\
\mathrm{ppm}\end{array}$ & $\begin{array}{c}\mathrm{Fe} \\
\mathrm{ppm}\end{array}$ & $\begin{array}{c}\mathrm{K} \\
\mathrm{ppm}\end{array}$ & $\begin{array}{l}\mathrm{Mn} \\
\mathrm{ppm}\end{array}$ & $\begin{array}{c}\mathrm{Zn} \\
\mathrm{ppm}\end{array}$ & $\begin{array}{c}\mathrm{Cu} \\
\mathrm{ppm}\end{array}$ & $\begin{array}{c}\mathrm{P} \\
\mathrm{ppm}\end{array}$ \\
\hline \multirow{4}{*}{ C. cadmea } & 1 & 2,0169 & 300 & 3910 & 31270 & 1290 & 23000 & 65 & 37,5 & 21,5 & 2100 \\
\hline & 2 & 2,3444 & 225 & 1840 & 17170 & 1110 & 16500 & 237 & 39,5 & 19 & 1700 \\
\hline & 3 & 1,1191 & 350 & 1800 & 17505 & 2940 & 20750 & 94,5 & 46 & 26 & 4450 \\
\hline & 1 & 1,3833 & 175 & 2655 & 25140 & 650 & 12500 & 37 & 26 & 7,5 & 700 \\
\hline \multirow[t]{2}{*}{ C. aphrodisea } & 2 & 2,2592 & 325 & 3395 & 25045 & 3880 & 15250 & 107,5 & 33 & 14,5 & 750 \\
\hline & 3 & 1,1414 & 450 & 1600 & 16635 & 545 & 11000 & 43,5 & 45 & 22 & 950 \\
\hline \multirow[t]{2}{*}{ C. amaena } & 1 & 1,9419 & 225 & 1915 & 28095 & 480 & 15750 & 51 & 55 & 11,5 & 1470 \\
\hline & 1 & 2,2657 & 450 & 2310 & 36200 & 2700 & 8750 & 113,5 & 107 & 114,5 & 1300 \\
\hline \multirow{3}{*}{ C. lycia } & 2 & 1,4619 & 500 & 2610 & 11205 & 3165 & 6500 & 118 & 93,5 & 44 & 500 \\
\hline & 3 & 2,8814 & 450 & 2515 & 37405 & 3725 & 13750 & 104 & 70 & 49 & 1300 \\
\hline & 4 & 1,5095 & 575 & 3860 & 34610 & 1230 & 10000 & 56 & 26,5 & 10,5 & 1050 \\
\hline \multirow{4}{*}{ C. luschaniana } & 1 & 1,4243 & 275 & 1330 & 17055 & 915 & 8750 & 55 & 58,5 & 22,5 & 950 \\
\hline & 2 & 1,1122 & 375 & 1365 & 16480 & 855 & 10000 & 61,5 & 40 & 20 & 1050 \\
\hline & 3 & 1,3064 & 250 & 1725 & 22455 & 2250 & 11000 & 91,5 & 74,5 & 51,5 & 1100 \\
\hline & 4 & 0,4922 & 325 & 5800 & 35340 & 1490 & 5750 & 52 & 28,5 & 10 & 600 \\
\hline \multirow{3}{*}{ C. wagenitzii } & 1 & 2,4083 & 625 & 7300 & 23070 & 1280 & 7750 & 96 & 68 & 71 & 600 \\
\hline & 2 & 0,9655 & 1650 & 6655 & 7135 & 445 & 9250 & 25,5 & 25 & 20 & 350 \\
\hline & 3 & 0,6426 & 2000 & 14305 & 9890 & 1260 & 8000 & 45,5 & 23,5 & 19,5 & 400 \\
\hline \multirow{3}{*}{ C. tossiensis } & 1 & 2,6647 & 175 & 1855 & 22550 & 1805 & 15250 & 86,5 & 41 & 15 & 3000 \\
\hline & 2 & 0,9985 & 225 & 1265 & 16210 & 160 & 13500 & 14,5 & 19,5 & 9,5 & 1250 \\
\hline & 3 & 1,8141 & 225 & 6300 & 21430 & 2615 & 17000 & 172,5 & 53 & 29,5 & 1750 \\
\hline \multirow{3}{*}{ C. hieropolitana } & 1 & 1,5874 & 450 & 2480 & 23890 & 465 & 15000 & 26 & 34,5 & 10 & 750 \\
\hline & 2 & 1,8227 & 275 & 7450 & 38015 & 265 & 9500 & 50,5 & 38,5 & 14 & 1000 \\
\hline & 3 & 2,6221 & 325 & 3895 & 27080 & 295 & 8750 & 25 & 34 & 12,5 & 1900 \\
\hline \multirow{2}{*}{ C. antalyense } & 1 & 1,7396 & 350 & 2115 & 29230 & 490 & 24750 & 35 & 89 & 38,5 & 1900 \\
\hline & 2 & 1,0479 & 700 & 1955 & 30165 & 4845 & 22000 & 147 & 40 & 17 & 1450 \\
\hline
\end{tabular}

\section{Physical Analysis of Soils}

C. cadmea prefers sandy-slime type soil, two sample areas of $C$. aphrodisea is sandy-slime type soils and another sample area is slimy sand (Table 5). C. amaena is found in sandy-slime soil; slimy-clay, slimy sand, sandy-slime, slime types soils have been observed in localities of C. lycia, has large soil type tolerance.

Table 5. The physical properties of the soils in the localities of species

\begin{tabular}{cccccccc}
\hline \multirow{2}{*}{ Species r } & Locality & Sand & Clay & Dust & Soil & \multicolumn{2}{c}{ Color of Soil } \\
& & $\%$ & $\%$ & $\%$ & Type & Dry & Wet \\
\hline \multirow{2}{*}{ C. cadmea } & 1 & 79,33 & 10,62 & 10,05 & Sandy- slime & $2,5 \mathrm{Y}-6 / 2$ & $2,5 \mathrm{Y}-3 / 2$ \\
& 2 & 84,9 & 6,23 & 8,82 & Sandy- slime & $2,5 \mathrm{Y}-4 / 2$ & $5 \mathrm{YR}-1,7 / 1$ \\
& 3 & 72,4 & 13,44 & 14,16 & Sandy- slime & $10 \mathrm{YR}-6 / 4$ & $10 \mathrm{YR}-4 / 4$ \\
& 1 & 76,39 & 14,61 & 9 & Sandy- slime & $10 \mathrm{YR}-5 / 4$ & $5 \mathrm{YR}-2 / 2$ \\
C. aphrodisea & 2 & 89 & 4,2 & 6,8 & Slimy-sand & $10 \mathrm{YR}-6 / 4$ & $5 \mathrm{YR}-2 / 2$ \\
& 3 & 74 & 13,3 & 12,7 & Sandy- slime & $5 \mathrm{YR}-4 / 3$ & $10 \mathrm{R}-2 / 3$ \\
& 1 & 70,9 & 12,8 & 16,3 & Sandy- slime & $7,5 \mathrm{YR}-5 / 3$ & $5 \mathrm{YR}-2 / 4$ \\
& 1 & 41,17 & 41,08 & 17,75 & Slimy-clay & $5 \mathrm{YR}-5 / 4$ & $5 \mathrm{YR}-3 / 6$ \\
& 2 & 86,31 & 7,5 & 6,18 & Slimy-sand & $2,5 \mathrm{Y}-5 / 3$ & $10 \mathrm{YR}-3 / 2$
\end{tabular}




\begin{tabular}{|c|c|c|c|c|c|c|c|}
\hline & 4 & 48,91 & 13,21 & 37,87 & Slime & 7,5 YR - 4/3 & $7,5 \mathrm{YR}-3 / 3$ \\
\hline \multirow{4}{*}{$\begin{array}{c}\text { C. } \\
\text { luschaniana }\end{array}$} & 1 & 56,02 & 38,95 & 5,03 & Sandy-clay & $7,5 \mathrm{YR}-5 / 4$ & $2,5 \mathrm{YR}-2 / 4$ \\
\hline & 2 & 31,23 & 38,51 & 30,25 & Slimy-clay & $5 \mathrm{YR}-5 / 6$ & $2,5 \mathrm{YR}-3 / 4$ \\
\hline & 3 & 41,47 & 32,35 & 26,17 & Slimy-clay & $5 \mathrm{YR}-4 / 4$ & $2,5 \mathrm{YR}-2 / 4$ \\
\hline & 4 & 43,75 & 33,57 & 22,68 & Slimy-clay & $7,5 \mathrm{YR}-6 / 6$ & $7,5 \mathrm{YR}-5 / 8$ \\
\hline \multirow{3}{*}{ C. wagenitzii } & 1 & 42,57 & 47,12 & 10,31 & Slimy-clay & $5 \mathrm{YR}-3 / 6$ & $7,5 R-3 / 6$ \\
\hline & 2 & 71,2 & 18,86 & 9,93 & Sandy- slime & $5 \mathrm{YR}-4 / 8$ & $10 \mathrm{R}-3 / 4$ \\
\hline & 3 & 59,71 & 25,45 & 14,84 & Sandy-clay & $5 \mathrm{YR}-4 / 6$ & $5 \mathrm{YR}-3 / 6$ \\
\hline \multirow{3}{*}{ C. tossiensis } & 1 & 87,91 & 4,03 & 8,06 & Slimy-sand & $10 \mathrm{YR}-5 / 2$ & $10 \mathrm{YR}-3 / 1$ \\
\hline & 2 & 48,58 & 39,08 & 12,34 & Slimy-clay & $10 \mathrm{YR}-5 / 3$ & $10 \mathrm{YR}-3 / 4$ \\
\hline & 3 & 72,46 & 15,17 & 12,37 & $\begin{array}{l}\text { Sandy-clay } \\
\text { Slime }\end{array}$ & $10 \mathrm{YR}-5 / 3$ & $10 \mathrm{YR}-4 / 4$ \\
\hline \multirow{3}{*}{$\begin{array}{c}C . \\
\text { hieropolitana }\end{array}$} & 1 & 64,24 & 19,54 & 16,22 & $\begin{array}{l}\text { Sandy-clay } \\
\text { Slime }\end{array}$ & $10 \mathrm{YR}-6 / 3$ & $5 \mathrm{YR}-4 / 3$ \\
\hline & 2 & 40,52 & 8,89 & 50,6 & Dusty-slime & $2,5 \mathrm{Y}-8 / 2$ & $10 \mathrm{YR}-7 / 2$ \\
\hline & 3 & 69,54 & 15,53 & 14,93 & $\begin{array}{l}\text { Sandy-clay } \\
\text { Slime }\end{array}$ & $2,5 Y-7 / 3$ & 7,5 YR $-4 / 3$ \\
\hline \multirow{2}{*}{ C. antalyense } & 1 & 41,46 & 34,48 & 24,06 & Slimy-clay & $10 \mathrm{YR}-3 / 3$ & $10 \mathrm{YR}-2 / 2$ \\
\hline & 2 & 38,82 & 38,19 & 22,98 & Slimy-clay & $7,5 \mathrm{YR}-4 / 3$ & $7,5 \mathrm{YR}-3 / 4$ \\
\hline
\end{tabular}

Soil types of distribution areas of C. luscahaniana are $75 \%$ of slimy-clay and $25 \%$ sandy clay. $C$. wagenitzii prefers slimy-clay, sandy-slime, and sandy clay type soils. $C$. tossiensis distributes in slimy-sand, slimy-clay, and sandy clay-loam type soils. Two sample areas of $C$. hieropolitana are sandy clay-loam type soils and in the other locality, soil type is dusty slime. Soil type of both two localities of $C$. anatlyense is slimy-clay.

Soil type rates of Phalolepis section are $30.7 \%$ of sandy-slime, $30.7 \%$ of slimy-clay, $11.6 \%$, of slimy-sand, $11.6 \%$ of sandy clay-loam, $7.8 \%$ of sandy-clay, $3.8 \%$ of slime and $3.8 \%$ of dusty-slime.

\section{Chemical analysis of soils}

Table 6 shows the chemical properties of the soils in the distribution areas of the species. C. cadmea spreads in slightly acidic and alkaline soil has large tolerance on the amount of $\mathrm{CaCO}_{3}$. Besides, first sample area of $C$. cadmea has the high rate of $\mathrm{P}_{2} \mathrm{O}_{5} . C$. aphrodisia prefers neutral and lightly alkaline soil like $C$. cadmea has tolerance to the $\mathrm{CaCO}_{3}$. Soil type of distribution area of C. amaena is neutral and without lime. C. lycia and $C$. luschaniana grow in limestone main rocks, they prefer high lime and alkaline soils. An also fourth sample area of $C$. lycia has the high rate of K. C. wagenitzii grows in lightly alkaline soils and localities of the species have the high rate of $\mathrm{Mg} . C$. tossiensis has a high tolerance for the soil type, prefers without lime or low lime soils.

C. hieropolitana grows on marl, lime, travertine bedrock so prefers alkaline and lime soils. C. antalyense prefers neutral, low amounts of lime soils, in the present study first sample area has the high rate of organic compounds. 
Table 6. The chemical properties of soils in the area of distribution of species

\begin{tabular}{|c|c|c|c|c|c|c|c|c|c|c|c|c|c|c|c|}
\hline Species & Locality & $\begin{array}{c}\mathrm{pH} \\
1 / 2,5 \\
\end{array}$ & $\begin{array}{c}\text { Salt } \\
\mathrm{ms} / \mathrm{cm}\end{array}$ & $\begin{array}{c}\text { Lime } \\
\%\end{array}$ & $\begin{array}{c}\text { Organic } \\
\%\end{array}$ & $\begin{array}{l}\mathrm{N} \\
\%\end{array}$ & $\begin{array}{c}\mathrm{Na} \\
\mathrm{ppm}\end{array}$ & $\begin{array}{c}\mathrm{Mg} \\
\mathrm{ppm}\end{array}$ & $\begin{array}{c}\mathrm{Ca} \\
\mathrm{ppm}\end{array}$ & $\begin{array}{c}\mathrm{Fe} \\
\mathrm{ppm}\end{array}$ & $\begin{array}{c}\mathrm{K} \\
\mathrm{ppm}\end{array}$ & $\begin{array}{c}\mathrm{Mn} \\
\mathrm{ppm}\end{array}$ & $\begin{array}{c}\mathrm{Zn} \\
\mathrm{ppm}\end{array}$ & $\begin{array}{c}\mathrm{Cu} \\
\mathrm{ppm}\end{array}$ & $\begin{array}{l}\mathrm{P}_{2} \mathrm{O}_{5} \\
\text { ppm }\end{array}$ \\
\hline \multirow{4}{*}{ C. cadmea } & 1 & 8,32 & 0,47 & 0,45 & 1,15 & 0,0186 & 15 & 47 & 3285 & 1,2 & 70 & 6,2 & 1,01 & 0,71 & 82,82 \\
\hline & 2 & 6,52 & 0,32 & 0 & 2,49 & 0,0497 & 30 & 73 & 946 & 1,1 & 51 & 10,3 & 1,01 & 0,2 & 16,16 \\
\hline & 3 & 8,64 & 0,3 & 55,9 & 0 & 0,0489 & 30 & 70 & 7049 & 0,4 & 71 & 6,8 & 1,11 & 0,3 & 5,05 \\
\hline & 1 & 7,95 & 0,37 & 0 & 0,98 & 0,028 & 21 & 1751 & 1569 & 1,4 & 93 & 4,8 & 0,82 & 0,21 & 5,15 \\
\hline \multirow[t]{2}{*}{ C. aphrodisea } & 2 & 7,45 & 0,28 & 0,15 & 0,4 & 0,0113 & 25 & 191 & 1546 & 1,1 & 56 & 5,9 & 1,31 & 0,4 & 16,16 \\
\hline & 3 & 8,59 & 0,24 & 44,5 & 0 & 0,047 & 21 & 2470 & 6586 & 1,3 & 101 & 0,7 & 0,74 & 0,42 & 37,1 \\
\hline C. amena & 1 & 7,36 & 0,47 & 0 & 2,87 & 0,0578 & 31 & 190 & 3005 & 1,1 & 296 & 7,2 & 4,18 & 0,82 & 74,46 \\
\hline \multirow{4}{*}{ C. lycia } & 1 & 8,26 & 0,41 & 44,5 & 2,02 & 0,2045 & 51 & 204 & 7781 & 0,6 & 416 & 9,4 & 1,13 & 0,93 & 18,54 \\
\hline & 2 & 8,09 & 0,34 & 0,9 & 0 & 0,0644 & 52 & 331 & 5673 & 0,4 & 98 & 6 & 1,13 & 0,62 & 21,63 \\
\hline & 3 & 7,98 & 0,44 & 39,2 & 1,33 & 0,3746 & 21 & 334 & 7631 & 1,2 & 165 & 3,2 & 0,93 & 0,62 & 18,54 \\
\hline & 4 & 8,21 & 0,72 & 25 & 11,33 & 0,5546 & 74 & 366 & 7917 & 1,7 & 1525 & 15,2 & 0,84 & 8,51 & 18,9 \\
\hline \multirow{4}{*}{ C. luschaniana } & 1 & 8,23 & 0,29 & 6,96 & 12,07 & 0,4188 & 37 & 581 & 8175 & 0,6 & 783 & 1,3 & 1,07 & 0,75 & 24,61 \\
\hline & 2 & 8,39 & 0,26 & 13,04 & 2,59 & 0,2657 & 37 & 296 & 8055 & 0,9 & 411 & 3,3 & 0,74 & 0,74 & 22,05 \\
\hline & 3 & 8,22 & 0,3 & 10,1 & 3,66 & 0,3265 & 53 & 262 & 8140 & 0,8 & 318 & 2,3 & 0,95 & 0,74 & 19,08 \\
\hline & 4 & 8,64 & 0,4 & 54,4 & 0,93 & 0,036 & 31 & 427 & 7381 & 0,5 & 258 & 0,7 & 0,72 & 10,61 & 27,81 \\
\hline \multirow{3}{*}{ C. wagenitzii } & 1 & 8,1 & 0,11 & 0 & 1,08 & 0,0201 & 251 & 3019 & 1745 & 0,5 & 238 & 10,2 & 1,16 & 0,65 & 6,45 \\
\hline & 2 & 7,94 & 0,36 & 0 & 0,58 & 0,0098 & 85 & 2360 & 746 & 0,3 & 97 & 6,6 & 0,91 & 0,34 & 5,7 \\
\hline & 3 & 8,22 & 0,81 & 0 & 0,28 & 0,0371 & 334 & 2639 & 1310 & 0,7 & 138 & 15,8 & 0,74 & 0,21 & 11,66 \\
\hline \multirow{3}{*}{ C. tossiensis } & 1 & 8,43 & 0,43 & 5,84 & 1,8 & 0,0693 & 25 & 137 & 6420 & 1 & 55 & 22,1 & 1,14 & 0,61 & 5,05 \\
\hline & 2 & 6,25 & 0,2 & 0 & 0,27 & 0,1156 & 36 & 244 & 1265 & 0,8 & 98 & 10,7 & 1,01 & 0,62 & 23,69 \\
\hline & 3 & 8,41 & 0,36 & 2,71 & 0,99 & 0,0789 & 26 & 112 & 7434 & 0,8 & 82 & 3,5 & 1,55 & 0,52 & 5,15 \\
\hline \multirow{3}{*}{ C. hieropolitana } & 1 & 8,22 & 0,39 & 28,4 & 1,27 & 0,177 & 25 & 215 & 6923 & 0,8 & 304 & 0,9 & 1,01 & 0,61 & 23,23 \\
\hline & 2 & 8,68 & 0,49 & 71,9 & 1,59 & 0,0419 & 21 & 214 & 7150 & 1,4 & 57 & 1,4 & 0,94 & 0,73 & 21,84 \\
\hline & 3 & 8,35 & 0,53 & 29,8 & 1,67 & 0,0223 & 32 & 147 & 7239 & 0,6 & 171 & 3,7 & 1,5 & 0,64 & 32,1 \\
\hline \multirow{2}{*}{ C. antalyense } & 1 & 7,45 & 0,13 & 2,19 & 12,95 & 0,7206 & 69 & 248 & 8000 & 1,8 & 297 & 16 & 1,38 & 1,17 & 9,54 \\
\hline & 2 & 7,65 & 0,58 & 0,5 & 1,92 & 0,1943 & 47 & 207 & 6259 & 1,2 & 287 & 5,6 & 1,35 & 0,62 & 16,64 \\
\hline
\end{tabular}




\section{Statistical Analysis}

In the analyses of the amount of the nutrient elements of the roots, the classification success have been found $92.3 \%$. One sample area of $C$. aphrodisea passed to C. lycia, one sample area of $C$. luschaniana passed to $C$. wagenitzii, other plant species stayed in their own groups (Fig. 2). First, two discrimination functions include $71,8 \%$ of all variance. In separation analyses of amounts of the nutrient elements of the roots, in the first function $\mathrm{Mn}, \mathrm{P}, \mathrm{Ca}$ and $\mathrm{N}$ are the most important variants, and in the second function $\mathrm{Ca}, \mathrm{Mn}, \mathrm{Zn}$, and $\mathrm{P}$ (Table 7).

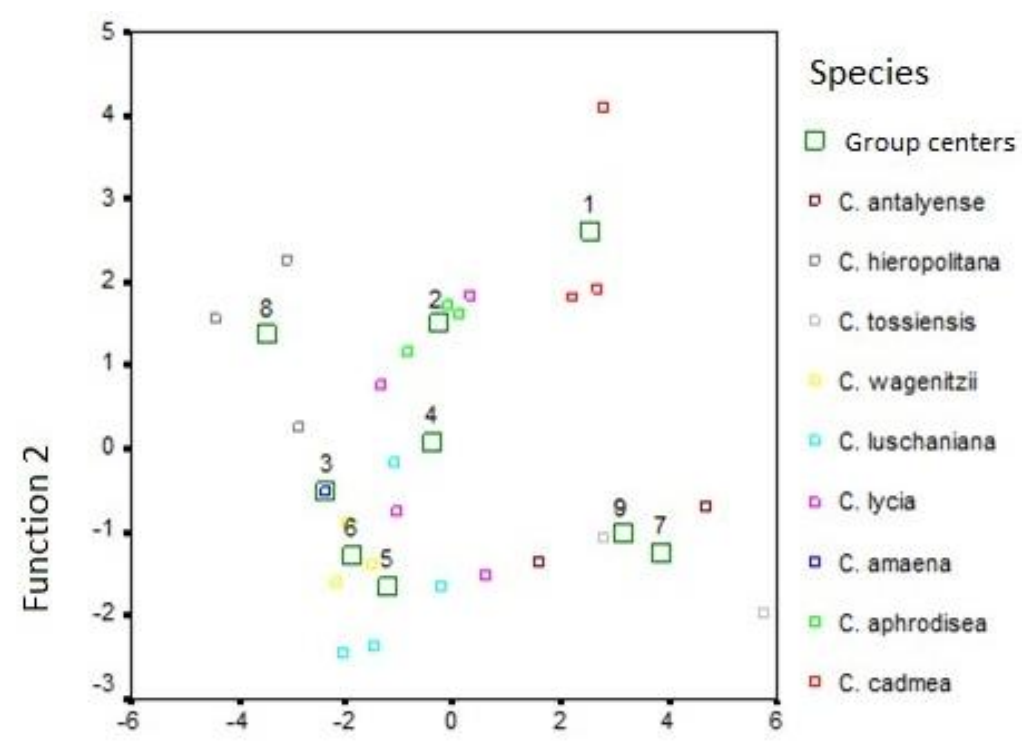

\section{Function 1}

Figure 2. The graphical representation of the discriminant analysis of amount of nutrient element analyses of roots

The classification analysis success of amounts of nutrient elements of the stems is 92.3\%. One sample area of $C$. cadmea passed to $C$. tossiensis, one sample area of $C$. hieropolitana passed to C. lycia, other plant species stayed in their own groups (Fig. 3). First, two functions of Table 8 explain the $77,1 \%$ of the variations. In separation analyses of amounts of the nutrient elements of the stems, in the first function $\mathrm{Na}, \mathrm{N}, \mathrm{P}$, and $\mathrm{Cu}$ are the most important variants, and in the second function $\mathrm{N}, \mathrm{Zn}, \mathrm{Fe}$, and $\mathrm{K}$.

Table 7. The results of discrimination of amount of nutrient elements of the roots

\begin{tabular}{|c|c|c|c|c|c|c|c|c|}
\hline Function & $\begin{array}{c}\text { Core } \\
\text { value }\end{array}$ & $\begin{array}{c}\text { Variance } \\
(\%)\end{array}$ & $\begin{array}{c}\text { Total } \\
(\%)\end{array}$ & $\begin{array}{c}\text { Kanon. } \\
\text { Korelas }\end{array}$ & $\begin{array}{c}\text { Wilks' } \\
\text { Lambda }\end{array}$ & Khi-Kare & SD & $\begin{array}{l}\text { Severity } \\
\text { level }\end{array}$ \\
\hline 1 & 8,408 & 51,7 & 51,7 &, 945 &, 001 & 102,391 & 80 &, 047 \\
\hline 2 & 3,279 & 20,1 & 71,8 &, 875 &, 013 & 67,646 & 63 &, 322 \\
\hline 3 & 2,471 & 15,2 & 87,0 &, 844 &, 054 & 45,113 & 48 &, 592 \\
\hline 4 &, 754 & 4,6 & 91,6 &, 656 &, 189 & 25,823 & 35 &, 871 \\
\hline 5 &, 611 & 3,8 & 95,4 &, 616 &, 332 & 17,113 & 24 &, 844 \\
\hline 6 &, 550 & 3,4 & 98,7 &, 596 &, 534 & 9,720 & 15 &, 837 \\
\hline 7 &, 190 & 1,2 & 99,9 &, 400 &, 828 & 2,925 & 8 &, 939 \\
\hline 8 &, 015 &, 1 & 100,0 &, 120 &, 986 &, 225 & 3 &, 973 \\
\hline
\end{tabular}


Standardized Separation Function Coefficients

\begin{tabular}{|c|c|c|c|c|c|c|c|c|}
\hline \multicolumn{9}{|c|}{ Function } \\
\hline & 1 & 2 & 3 & 4 & 5 & 6 & 7 & 8 \\
\hline $\mathrm{N} \%$ & $-1,103$ & ,339 &, 113 & $-1,010$ & ,605 &,- 523 & $-1,063$ & ,009 \\
\hline $\mathrm{Na}$ ppm &,- 418 & ,329 &,- 329 & ,547 & ,417 & 280 & ,961 & ,063 \\
\hline $\mathrm{Mg}$ ppm & ,003 & ,197 &,- 470 & ,886 &,- 510 & ,314 & ,383 & ,740 \\
\hline Ca ppm & $-1,201$ & ,626 & ,416 &,- 740 &,- 288 & ,016 & ,130 &, 125 \\
\hline Fe ppm &,- 361 & ,147 & ,085 & ,246 & 883 &,- 243 &,- 950 &,- 166 \\
\hline $\mathrm{K}$ ppm & 1,099 & ,409 & ,039 & ,298 &, 003 & 849 &,- 258 &,- 008 \\
\hline $\mathrm{Mn}$ ppm & 1,457 & ,599 &,- 017 & ,676 &,- 470 &,- 080 & 1,078 &,- 116 \\
\hline $\mathrm{Zn} \mathrm{ppm}$ &,- 660 &,- 530 & ,365 & ,009 &,- 268 & 243 &,- 054 &,- 164 \\
\hline $\mathrm{Cu}$ ppm &,- 106 &,- 347 & 892 & ,179 & ,093 &, 130 &,- 018 & 307 \\
\hline $\mathrm{P} \quad \mathrm{ppm}$ & 1,431 &,- 504 &,- 595 &,- 324 & ,212 &,- 507 & ,497 & 346 \\
\hline
\end{tabular}

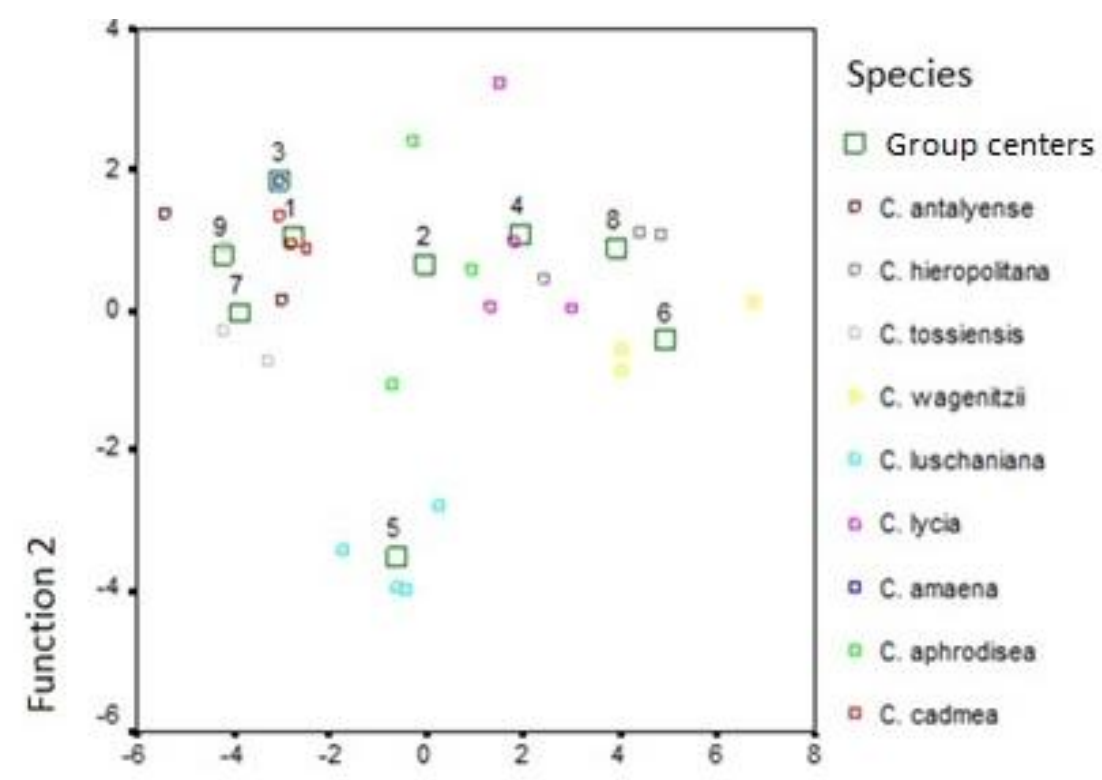

Function 1

Figure 3. The graphical representation of the discriminant analysis of amount of nutrient element analyses of stems

Table 8. The discriminate analysis results of amount of nutrient element analyses of stems

\begin{tabular}{|c|c|c|c|c|c|c|c|c|}
\hline Function & $\begin{array}{c}\text { Core } \\
\text { value }\end{array}$ & $\begin{array}{c}\text { Variance } \\
(\%)\end{array}$ & $\begin{array}{c}\text { Total } \\
(\%)\end{array}$ & $\begin{array}{c}\text { Kanon. } \\
\text { Korelas }\end{array}$ & $\begin{array}{c}\text { Wilks' } \\
\text { Lambda }\end{array}$ & Khi-Kare & SD & $\begin{array}{l}\text { Severity } \\
\text { level }\end{array}$ \\
\hline 1 & 14,596 & 60,8 & 60,8 &, 967 &, 000 & 118,585 & 80 &, 003 \\
\hline 2 & 3,927 & 16,3 & 77,1 &, 893 &, 007 & 76,006 & 63 &, 126 \\
\hline 3 & 2,652 & 11,0 & 88,2 &, 852 &, 037 & 51,289 & 48 &, 346 \\
\hline 4 & 1,198 & 5,0 & 93,1 &, 738 &, 133 & 31,213 & 35 &, 652 \\
\hline 5 & 1,096 & 4,6 & 97,7 &, 723 &, 293 & 19,004 & 24 &, 752 \\
\hline 6 &, 359 & 1,5 & 99,2 &, 514 &, 615 & 7,534 & 15 &, 941 \\
\hline 7 &, 173 &, 7 & 99,9 &, 384 &, 836 & 2,785 & 8 &, 947 \\
\hline 8 &, 020 &, 1 & 100,0 &, 140 &, 980 &, 307 & 3 &, 959 \\
\hline
\end{tabular}


Standardized Separation Function Coefficients

\begin{tabular}{|l|c|c|c|c|c|c|c|c|}
\hline \multicolumn{9}{|c|}{ Function } \\
\hline & 1 & 2 & 3 & 4 & 5 & 6 & 7 & 8 \\
\hline $\mathrm{N} \%$ & 1,764 & 1,565 &, 217 &, 031 &,- 016 &,- 553 &, 054 &,- 243 \\
\hline $\mathrm{Na} \mathrm{ppm}$ & 1,834 &, 444 &, 144 &, 602 &, 123 &, 082 &, 225 &,- 191 \\
\hline Mg ppm &, 486 &, 433 &, 040 &, 199 &, 835 &, 151 &, 142 &, 614 \\
\hline Ca ppm &, 160 &,- 244 &,- 294 &,- 844 &,- 368 &, 518 &, 106 &,- 501 \\
\hline Fe ppm &, 060 &, 873 &, 059 &, 303 &,- 445 &, 127 &, 177 &, 772 \\
\hline K ppm &,- 648 &, 789 &,- 598 &, 164 &,- 118 &, 420 &, 172 &, 006 \\
\hline Mn ppm &,- 425 &,- 337 &, 969 &, 010 &, 051 &, 257 &, 106 &,- 410 \\
\hline Zn ppm &, 336 & $-1,271$ &,- 320 &, 345 &, 270 &, 532 &, 591 &,- 432 \\
\hline Cu ppm &,- 649 &,- 184 &,- 227 &,- 480 &,- 534 &,- 405 &, 272 &, 607 \\
\hline P ppm & $-1,017$ &,- 137 &, 431 &, 128 &, 865 &,- 438 &, 269 &, 070 \\
\hline
\end{tabular}

The classification analysis success of amounts of nutrient elements of the leaves have been found $88.5 \%$. One sample area of $C$. aphrodisea passed to C. luschaniana, one sample area of $C$. luschaniana and $C$. hieropolitana passed to $C$. aphrodisea, other plant species stayed in their own groups (Fig. 4).

First, two separation functions explain the $85,3 \%$ of all variations. In the first function $\mathrm{Mn}, \mathrm{N}, \mathrm{K}$ and $\mathrm{Cu}$, in the second function $\mathrm{Zn}, \mathrm{Cu}, \mathrm{Ca}$ and $\mathrm{Mg}$ have provided the biggest contribution (Table 9).

The discrimination analysis success of chemical and physical soil $(0-10 \mathrm{~cm})$ contents of the species is $96.2 \%$. One sample area of $C$. hieropolitana passed to $C$. lycia, other plant species stayed in their own groups (Fig. 5).

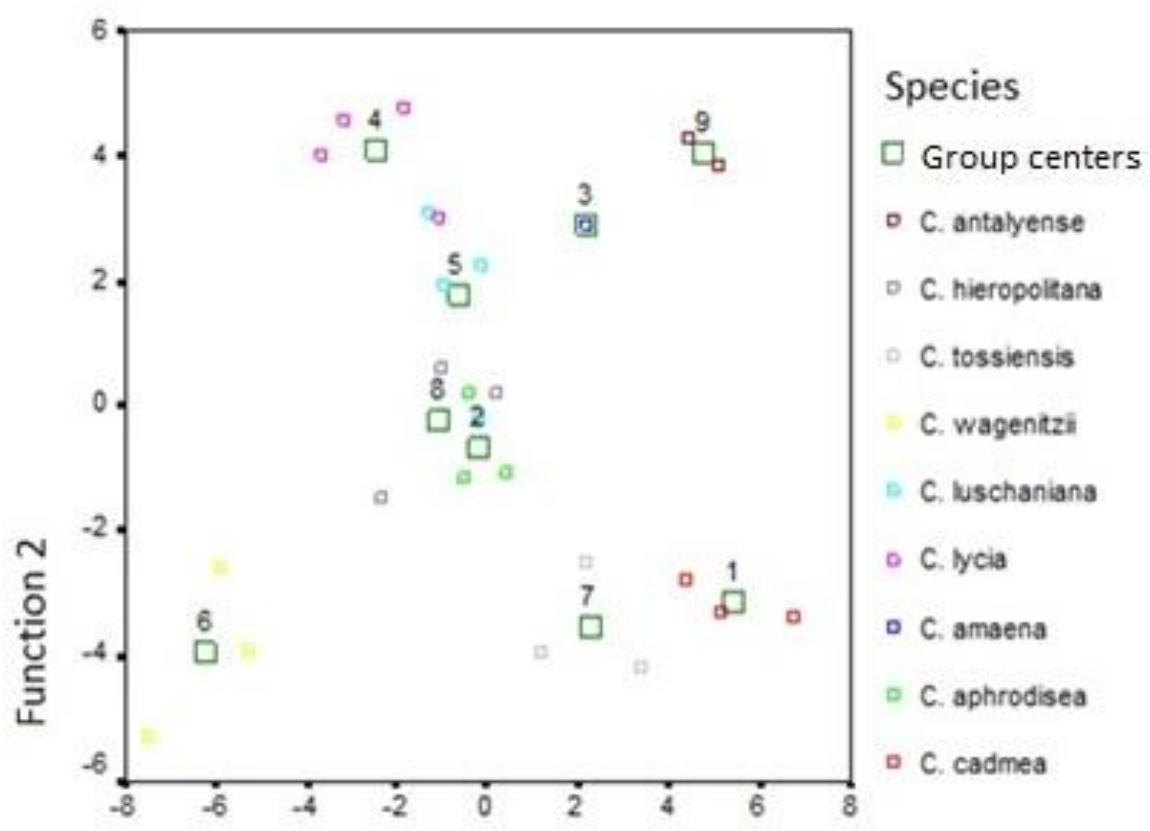

\section{Function 1}

Figure 4. The graphical representation of the discriminant analysis of amount of nutrient element analyses of leaves 
Table 9. The discriminate analysis results of amount of nutrient element analyses of leaves

\begin{tabular}{|c|c|c|c|c|c|c|c|c|}
\hline Function & $\begin{array}{c}\text { Core } \\
\text { value }\end{array}$ & $\begin{array}{c}\text { Variance } \\
(\%)\end{array}$ & $\begin{array}{c}\text { Total(\% } \\
)\end{array}$ & $\begin{array}{c}\text { Kanon. } \\
\text { Korelas }\end{array}$ & $\begin{array}{c}\text { Wilks' } \\
\text { Lambda }\end{array}$ & Khi-Kare & SD & $\begin{array}{l}\text { Severity } \\
\text { level }\end{array}$ \\
\hline 1 & 17,631 & 47,7 & 47,7 &, 973 &, 000 & 133,598 & 80 &, 000 \\
\hline 2 & 13,893 & 37,6 & 85,3 &, 966 &, 003 & 88,264 & 63 &, 020 \\
\hline 3 & 3,488 & 9,4 & 94,8 &, 882 &, 050 & 46,400 & 48 &, 539 \\
\hline 4 &, 821 & 2,2 & 97,0 &, 671 &, 225 & 23,127 & 35 &, 938 \\
\hline 5 &, 698 & 1,9 & 98,9 &, 641 &, 410 & 13,838 & 24 &, 950 \\
\hline 6 &, 331 &, 9 & 99,8 &, 499 &, 695 & 5,634 & 15 &, 985 \\
\hline 7 &, 044 &, 1 & 99,9 &, 204 &, 925 & 1,200 & 8 &, 997 \\
\hline 8 &, 035 &, 1 & 100,0 &, 185 &, 966 &, 540 & 3 &, 910 \\
\hline
\end{tabular}

Standardized Separation Function Coefficients

\begin{tabular}{|l|c|c|c|c|c|c|c|c|c|}
\hline \multicolumn{9}{|c|}{ Function } \\
\hline & 1 & 2 & 3 & 4 & 5 & 6 & 7 & 8 \\
\hline $\mathrm{N} \%$ & $-1,026$ &,- 756 &,- 010 &, 684 &, 215 &, 899 &, 275 &,- 305 \\
\hline $\mathrm{Na} \mathrm{ppm}$ &,- 640 & 1,342 & 1,297 &, 193 &, 415 &,- 119 &,- 283 &,- 486 \\
\hline $\mathrm{Mg} \mathrm{ppm}$ &,- 297 & $-1,766$ &,- 199 &, 212 &,- 212 &,- 074 &, 398 &, 739 \\
\hline $\mathrm{Ca} \mathrm{ppm}$ &, 315 & 2,504 &, 537 &, 364 &, 559 &,- 275 &,- 705 &, 076 \\
\hline Fe ppm &,- 620 &, 311 &,- 510 &,- 412 &,- 570 &, 751 &, 264 &, 532 \\
\hline $\mathrm{K} \mathrm{ppm}$ &, 923 &,- 519 &, 459 &, 096 &,- 493 &, 134 &, 008 &, 058 \\
\hline $\mathrm{Mn} \mathrm{ppm}$ & 1,177 &, 240 &, 610 &,- 341 &, 715 &,- 462 &,- 378 &,- 594 \\
\hline $\mathrm{Zn} \mathrm{ppm}$ &, 276 & 3,350 &, 655 &, 683 &, 262 &,- 696 & 1,032 &,- 033 \\
\hline $\mathrm{Cu} \mathrm{ppm}$ &,- 739 & $-2,700$ &,- 039 & $-1,064$ &,- 222 &, 513 &,- 606 &, 326 \\
\hline $\mathrm{P} \mathrm{ppm}$ &, 654 &,- 332 &, 123 &,- 065 &, 831 &,- 276 &, 061 &, 211 \\
\hline
\end{tabular}

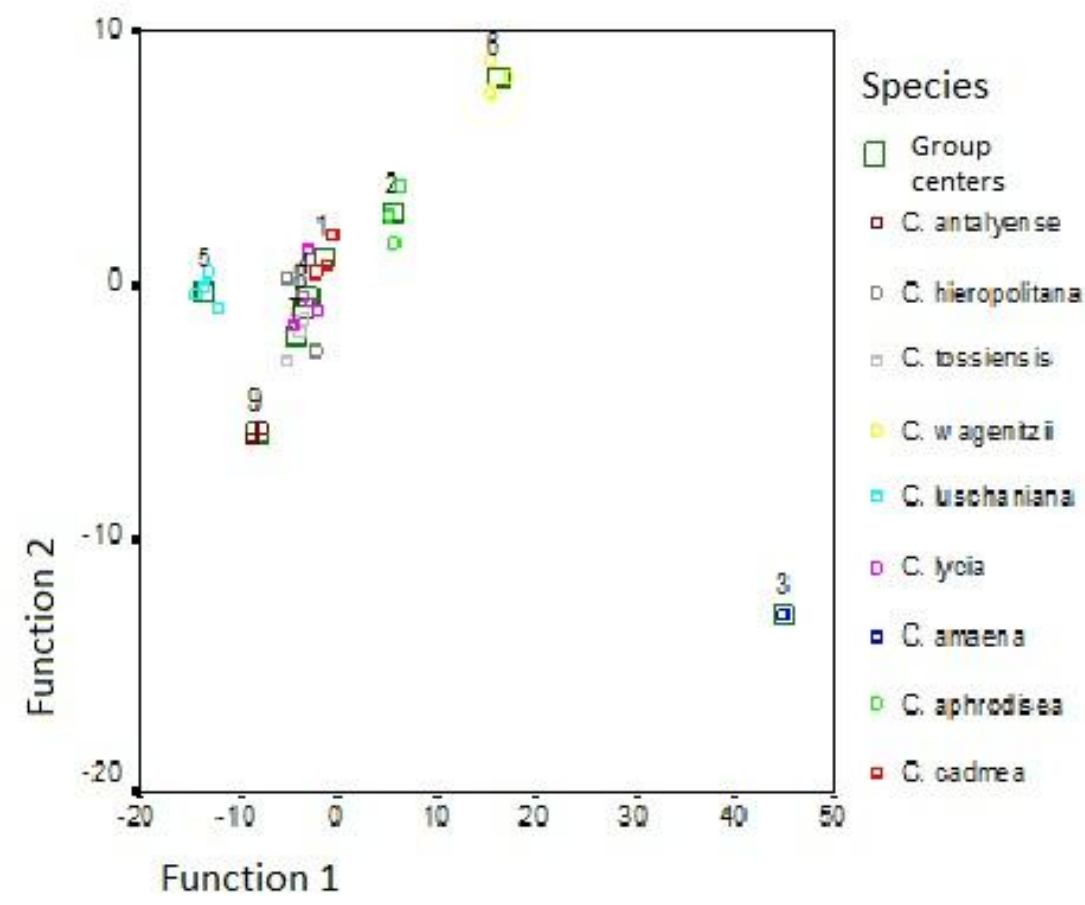

Figure 5. The graphical representation of the discriminant analysis of chemical and physical properties of the soils $(0-10 \mathrm{~cm})$ of the distribution localities of the species 
First, two separation functions explain the $92,4 \%$ of all variations. In the first function $\mathrm{Ca}^{++}, \mathrm{N}$, the organic compound, and $\mathrm{Zn}^{++}$, in the second function $\mathrm{Ca}^{++}, \mathrm{pH}$, sand and $\mathrm{K}$ amounts are important (Table 10).

Table 10. The discriminate analysis results of chemical and physical properties of the soils $(0-10 \mathrm{~cm})$ of the distribution localities of the species

\begin{tabular}{|c|c|c|c|c|c|c|c|c|}
\hline Function & $\begin{array}{c}\text { Core } \\
\text { value }\end{array}$ & $\begin{array}{c}\text { Variance } \\
(\%)\end{array}$ & $\begin{array}{c}\text { Total(\% } \\
)\end{array}$ & $\begin{array}{c}\text { Kanon. } \\
\text { Korelas }\end{array}$ & $\begin{array}{c}\text { Wilks' } \\
\text { Lambda }\end{array}$ & Khi-Kare & SD & $\begin{array}{l}\text { Severity } \\
\text { level }\end{array}$ \\
\hline 1 & 227,09 & 82,0 & 82,0 &, 998 &, 000 & 194,444 & 128 &, 000 \\
\hline 2 & 28,653 & 10,4 & 92,4 &, 983 &, 000 & 126,572 & 105 &, 075 \\
\hline 3 & 13,331 & 4,8 & 97,2 &, 964 &, 001 & 84,202 & 84 &, 473 \\
\hline 4 & 4,121 & 1,5 & 98,7 &, 897 &, 017 & 50,922 & 65 &, 899 \\
\hline 5 & 1,635 &, 6 & 99,3 &, 788 &, 087 & 30,505 & 48 &, 977 \\
\hline 6 & 1,063 &, 4 & 99,7 &, 718 &, 230 & 18,394 & 33 &, 981 \\
\hline 7 &, 563 &, 2 & 99,9 &, 600 &, 474 & 9,344 & 20 &, 979 \\
\hline 8 &, 351 &, 1 & 100,0 &, 510 &, 740 & 3,762 & 9 &, 926 \\
\hline
\end{tabular}

Standardized Separation Function Coefficients

\begin{tabular}{|l|c|c|c|c|c|c|c|c|}
\hline \multicolumn{9}{|c|}{ Function } \\
\hline & 1 & 2 & 3 & 4 & 5 & 6 & 7 & 8 \\
\hline $\mathrm{pH}$ & 2,652 & 1,691 &, 088 &, 043 &,- 326 &, 323 &,- 488 &, 807 \\
\hline Salt &,- 060 &,- 822 & $-1,109$ &,- 166 &,- 021 &, 023 &, 700 &, 007 \\
\hline Lime & 2,823 &, 508 & 1,788 &, 688 &, 043 &, 188 &, 539 &, 547 \\
\hline Dust & 1,913 &, 441 &,- 954 & 1,552 &,- 010 &,- 495 &, 282 &,- 748 \\
\hline Sand & 2,334 &, 942 & 1,156 & 1,073 &, 466 &, 055 &, 109 &,- 288 \\
\hline Org. comp. & $-3,724$ &, 004 &,- 614 &, 141 & $-1,787$ & $-1,107$ &, 229 &, 204 \\
\hline $\mathrm{N}$ & 3,976 &, 400 & $-1,523$ &, 643 & 1,132 & 1,045 &, 089 & 1,082 \\
\hline $\mathrm{Na}^{+}$ &,- 033 &, 826 &, 322 &, 496 &,- 100 &,- 089 &, 186 &, 252 \\
\hline $\mathrm{Mg}^{++}$ & 2,409 &, 121 &,- 827 &,- 314 &, 051 &, 219 &, 074 &,- 414 \\
\hline $\mathrm{Ca}^{++}$ & $-5,822$ & $-2,083$ &,- 480 &,- 491 &, 207 &,- 234 &, 189 & $-1,191$ \\
\hline $\mathrm{Fe}^{++}$ &,- 739 &,- 744 & 1,847 & $-1,637$ &,- 465 &, 510 &, 187 &, 230 \\
\hline $\mathrm{K}^{+}$ & 2,098 &, 932 & 2,160 &, 702 &, 831 &, 740 &,- 352 &,- 441 \\
\hline $\mathrm{Mn}^{++}$ & 1,003 &,- 421 &,- 164 &,- 313 &, 904 &,- 616 &,- 040 &,- 117 \\
\hline $\mathrm{Zn}^{++}$ & 3,232 &,- 855 &,- 260 &,- 054 &,- 006 &, 128 &,- 001 &,- 057 \\
\hline $\mathrm{Cu}^{++}$ & $-1,042$ &,- 507 & $-1,096$ &,- 379 &, 060 &, 256 &,- 579 &, 041 \\
\hline $\mathrm{P}_{2} \mathrm{O}_{5}$ &, 481 &,- 178 &, 133 &, 830 &, 075 &,- 238 &,- 240 &, 342 \\
\hline
\end{tabular}

\section{Conclusion}

Because of limited distribution areas, population of these species are under risk of being extinct. Having narrow habitat let species in Phalolepis section be damaged by any natural or antropogenic activity. Tourism activities (Saklikent, Termessos), agriculture, road construction, grazing and building activities in the region are the most dangerous activities for future of these species populations. Conservation strategies must be performed immediately for species. 


\section{REFERENCES}

[1] Olson, D. M., Dinerstein, E. (1998): The Global 200: A Representation Approach to Conserving the Earth's Most Biologically Valuable Ecoregions.- Conservation Biology 12(3): 502-515.

[2] Mittermeier, R. A., Myers, N., Thomsen, J. B., da Fonseca, G. A. B., Olivieri, S. (1998): Biodiversity hotspots and major tropical wilderness areas: Approaches to setting conservation priorities.- Conservation Biology 12: 516-520.

[3] IUCN Species Survival Commission (2001): IUCN Red list categories. Version 3.1. IUCN. Gland, Switzerland and Cambridge, UK.

[4] Favarger, C. (1972): Endemism in the mountain floras of Europe. - In: Valentine, D.H. (ed.) Taxonomy, phytogeography and evolution. Academic Press, London and New York, 191-204.

[5] Gomez Campo, C., Bermudez de Castro, M., Cagiga, M.J., Sanchez Yelamo, M.D. (1984): Endemism in the Iberian Peninsula and Balearic Islands.- Webbia 38: 709-714.

[6] Rabinowitz, D., Cairns, S., Dillon, T. (1986): Seven forms of rarity and their frequency in the flora of the British Isles. In: Soulé, M. E. (ed.) Conservation biology: the science of scarcity and diversity. Sinauer, Sunderland, Massachusetts, USA, 182-204.

[7] Gaston, K. J., (1994): Rarity. - Chapman and Hall, London, UK.

[8] Norton, D.A., De Lange, P.J. (1998): Hebe paludosa (Scrophulariaceae) - a new combination for an endemic wetland Hebe from Westland, South Island, New Zealand. New Zealand Journal of Botany 36: 531-538.

[9] Wyatt, R. (1997): Reproductive ecology of granite outcrop plants from the south-eastern United States.- Journal of Royal Society of Western Australia 80: 123-129.

[10] Larson, D.W., Matthes, U., Kelly, P.E. (2000): Cliff ecology: pattern and process in cliff ecosystem. - Cambridge: Cambridge University Press.

[11] Hopper, S.D. (2000): How well do phylogenetic studies inform the conservation of Australian plants?.- Australian Journal of Botany 48: 321-328.

[12] Ekim, T., Koyuncu, M., Vural, M., Duman, H., Aytaç, Z., Adıgüzel, N. (2000): Türkiye Bitkileri Kırmızı Kitabı (Egrelti ve Tohumlu Bitkiler).- Türkiye Tabiatını Koruma Dernegi, Van Yüzüncü Yıl Üniversitesi Yayınları, Ankara.

[13] Brummitt, R. K. (2004): Report of the committee for spermatophyta.-Taxon 53(3): 813825.

[14] Kültür, Ş., Bona, M., Nath E. Ö. (2016): A new species of Centaurea (Asteraceae) from East Anatolia, Turkey.-Phytotaxa 247 (1): 85-91.

[15] Duberted, L. (1973): Türkiye Jeoloji Haritas1 (Izmir).- Maden Tetkik Arama Enstitüsü, 115.

[16] Jackson, M. L. (1962): Soil chemical analysis.-Prentice Holl vinc Englewood.

[17] Chapman, H. D., Pratt, F. P. (1961): Methods of analysis for soil plants and waters.University of California.

[18] Walkley, A., Black, I. A. (1934): An examination of the method for determining soil organic matter and a proposed modification of the chromic acid method.- Soil Science, 37: 29-38.

[19] Bouyoucus, C. J. (1962): Hydrometer method for making particle size analysis of soil. Agronomoy Journal 54: 5.

[20] Çepel, N. (1983): Orman Ekolojisi. - Istanbul University 3140, Istanbul.

[21] Kantarc1, D. (2000): Toprak ilmi. - Istanbul Üniversitesi Yayın No. 4261, Istanbul.

[22] Eruz, E. (1979): Toprak tuzlulugu ve bitkiler üzerindeki etkileri.- Istanbul Üniversitesi, Orman Fakültesi Dergisi 29(2): 112-120.

[23] Schöreder, D. (1972): Bodenkunde in Stichworten. - Verlag Ferdinant Hirt, Kiel.

[24] Tüzüner, A. (1990): Toprak ve su analiz laboratuarları El Kitabı. - Köy Hizmetleri Genel Müdürlügü, Ankara. 